\title{
Direct Observation of the Evolution of Plasmonic Heterostructured Nanowires
}

\author{
Bethany M. Hudak ${ }^{1}$, Yao-Jen Chang ${ }^{1}$, Guohua Li $^{1}$ and Beth S. Guiton ${ }^{1,2}$ \\ 1. Department of Chemistry, University of Kentucky, Lexington, KY 40506 \\ 2. Materials Science and Technology Division, Oak Ridge National Laboratory, Oak Ridge TN 37831
}

Metal-dielectric heterostructures have received much interest in recent years because of properties due to the localized surface plasmon resonances (LSPRs) at the metal/dielectric interfaces, such as subdiffraction limit waveguiding and colossally-enhanced photoemission. Noble metal nanoparticle chains have been fabricated using several top-down methods such as lithography and templating, but the bottom-up approach of a peapodded nanowire (NW) is attractive because the nanoparticles form spontaneously during synthesis and have the potential to be very well-defined in terms of size, spacing, shape, and interfacial orientation. Moreover, the NW oxide casing can protect the metal particles from oxidation, giving such plasmonic structures the potential to be made from a diverse range of metals, and with control over their dielectric environment.

The functionality of these hybrid materials depends critically on the nature of the metal/metal oxide interface, and the size, shape, orientation, and spacing of the metallic regions. Here we report the use of in situ transmission electron microscopy (TEM) techniques to elucidate the mechanisms by which metal/metal oxide NW heterostructures evolve, by replicating in the TEM the processing conditions used to form these structures. By observing the changing crystallography of the NWs directly during evolution, we bridge the gap between structure and functionality.

Core-shell and peapod nanowires are heterostructured nanowires with crystallographically distinct metal cores or metal nanoparticles embedded in a metal oxide nanowire casing. Several core-shell nanowire syntheses have been reported using a variety of techniques, such as chemical treatment of silica-coated nanoparticles [1], atomic layer deposition [2], and nanoparticle chain encapsulation [3], but a simple two-step synthesis using vapor-liquid-solid (VLS) nanowire growth and annealing can be performed to produce nanowires that form cores or "peas" in some known systems, spontaneously [4]. Hsieh et al. first published this synthetic technique in 2008 as a method to grow Au-in-Ga2O3 peapod NWs [5]. A schematic of the synthesis of Au-in-Ga2O3 peapod NWs is shown in Fig. 1(a). Core-shell and peapod formation occurs via secondary annealing steps after traditional VLS synthesis of homogeneous NWs; NWs develop a metal core within the oxide shell on first annealing, which splits into discrete linear gold segments when the temperature is increased; further annealing facilitates the transformation of linear segments to gold nanoparticles, with typical annealing times in the range of a few minutes to an hour or two [4]. Au-in- $\mathrm{Ga}_{2} \mathrm{O}_{3}$ provides a particularly nice model system, since it is known to spontaneously form particularly well-defined faceted gold cores or nanoparticles.

Shown in Fig. 1(b) is preliminary in situ data from a Au-in- $\mathrm{Ga}_{2} \mathrm{O}_{3}$ core-shell nanowire, grown by us using the synthesis reported by Ref. [5]. On annealing in the TEM, at temperatures expected to cause formation of a peapod structure, the gold head is unexpectedly seen to lose its conical tip, move into the wire, and agglomerate with the metal core to form a single encapsulated particle. Similar phenomena have since been observed in a number of other $\mathrm{Au}$-in- $\mathrm{Ga}_{2} \mathrm{O}_{3}$ nanowires, with initial observations indicating that wire diameter may be significant in determining heterostructure morphology [6]. 
References:

[1] S. E. Hunjadi and C. J. Murphy, J. Phys. Chem. B 110 (2006), p. 7226.

[2] Y. Qin et al, Nano Lett. 8 (2008), p. 3221.

[3] Y. Yao, G. S. Chaubey, and J. B. Wiley, J. Am. Chem. Soc. 134 (2012), p. 2450.

[4] P.-H. Chen et al, Nano Lett. 10 (2010), p. 3267.

[5] C.-H. Hsieh et al, Nano Lett. 8 (2008), p. 3081.

[6] The authors acknowledge funding from the Office of Basic Energy Sciences, Materials Sciences and Engineering Division, U.S. Department of Energy (BSG), and the Kentucky NSF EPSCoR program through the Center of Advanced Materials.

(a)

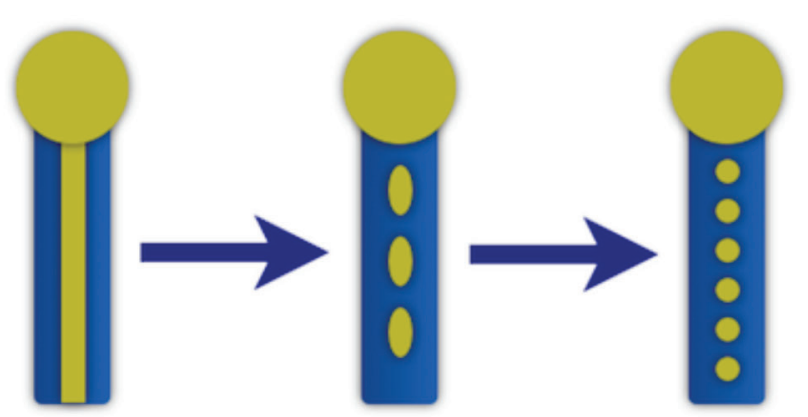

(b)

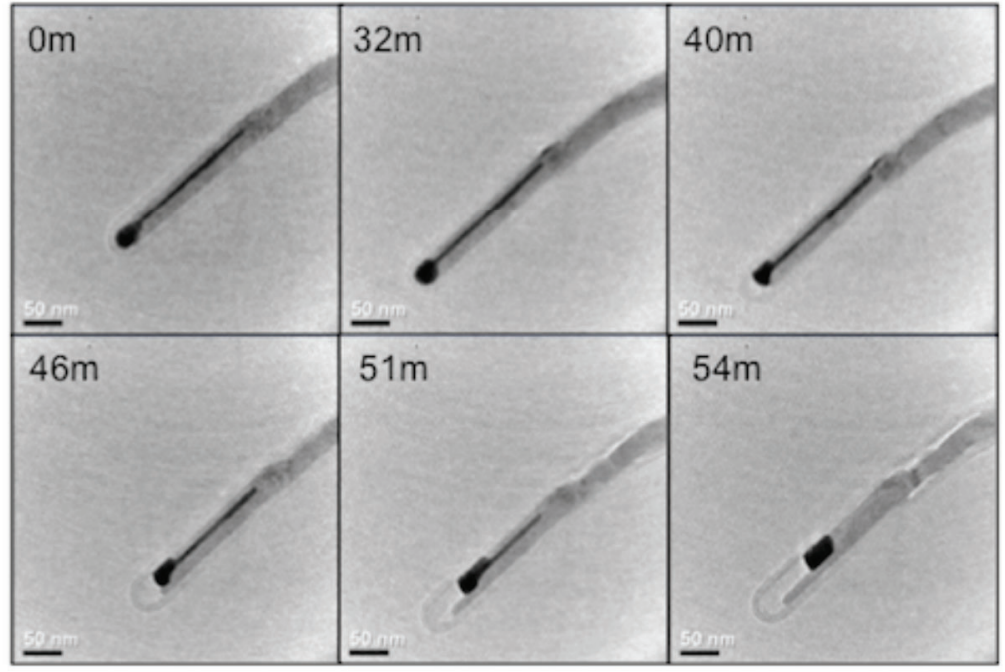

Figure 1. Metal/metal oxide nanowire heterostructure evolution. (a) Schematic showing the evolution from core-shell to peapod morphology reported for $\mathrm{Au}-\mathrm{in}-\mathrm{Ga}_{2} \mathrm{O}_{3}$. (b) TEM images of a $\mathrm{Au}-\mathrm{in}-\mathrm{Ga}_{2} \mathrm{O}_{3}$ core-shell nanowire evolving in situ. 